\title{
Combination of oxybutynin transdermal patch and heparinoid cream for long-term treatment of overactive bladder in elderly patients
}

Yoshihito Murakami ${ }^{1,2}$, Hiroshi Nagae ${ }^{3}$, Naomi Maehori ${ }^{3}$, Hidehisa Sekijima ${ }^{1}$ and Kazuya Ooi ${ }^{*}$

*Correspondence: Kazuya Ooi zooi@suzuka-u.ac.jp

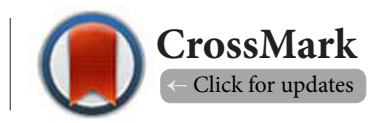

'Faculty of Pharmaceutical Sciences, Suzuka University of Medical Science, Suzuka, Mie, Japan.

${ }^{2}$ Sunai Pharmacy Co., Ltd., Kuwana, Mie, Japan.

${ }^{3}$ Nagae Prostate Care Clinic, Hamamatsu, Shizuoka, Japan.

\begin{abstract}
Background: Overactive bladder is an age-related urologic disease. An oxybutynin transdermal patch is used to treat overactive bladder; however, long-term use is not advised because of potential adverse reactions at the site of application. The present study aimed to investigate the advantages of administering a combination of heparinoid cream and oxybutynin transdermal patch treatment for overactive bladder in elderly patients.

Methods: Eight patients ( $>65$ years of age) with overactive bladder were included. The heparinoid cream was applied topically for 1 week, and skin hydration and transepidermal water loss were quantified before and after application. Thereafter, patients were treated with a combination of an oxybutynin transdermal patch and a heparinoid cream for 12 weeks, and the sites of application were monitored for adverse reactions. Clinical effects of the combinatorial treatment were assessed based on the overactive bladder symptom score.

Results: Application of the heparinoid cream did not decrease transepidermal water loss, but increased skin hydration and improved dryness. Oxybutynin transdermal patch therapy was not interrupted because no skin irritation was observed at the site of application for 12 weeks, and the overactive bladder symptom score decreased significantly $(\mathrm{p}<0.05)$

Conclusions: Combination treatment with an oxybutynin transdermal patch and a heparinoid cream improved treatment outcomes, suggesting that skin hydration with heparinoid cream is a promising treatment method to ensure appropriate use of oxybutynin transdermal patches in elderly patients with overactive bladders.
\end{abstract}

Keywords: Heparinoids, overactive bladder, skin care, transdermal patch

\section{Introduction}

The incidence of overactive bladder (OAB) has increased in Japan because of rapid aging of the population [1]. As frequent urination, a major symptom of $O A B$, decreases the quality of life of patients with $O A B$ [2], therapeutic drugs including muscarinic antagonists and $\beta 3$-adrenoceptor agonists are administered clinically to improve bladder urine retention. Currently, selective muscarinic antagonists are used as first-line drugs to treat $O A B[3]$ and have been developed as an alterna- tive to oral administration of oxybutynin in order to enhance pharmacotherapeutic tolerance.

The oxybutynin transdermal patch is a sustained-release drug formulation containing oxybutynin hydrochloride [4]. Production of $\mathrm{N}$-desethyloxybutynin (DEO), an active metabolite of oxybutynin, is lower with transdermal patches than with oral formulations because oxybutynin is absorbed through the skin, thereby avoiding the hepatic first-pass effect [5]. Anticholinergic effects of DEO increase therapeutic 
Murakami et al. Dermatology Aspects 2018,

efficacy; however, they also cause side effects including dry mouth and constipation [6]. Use of oxybutynin transdermal patches maintains DEO at low levels during OAB treatment without affecting the activities of daily living [7]. Long-term use of oxybutynin transdermal patches is associated with an increased incidence of skin irritation at the site of application [8]. Therefore, skin conditions of patients with OAB should be evaluated before applying oxybutynin transdermal patches.

The skin of elderly individuals is often dry because of reduced sebum secretion and hydration of the stratum corneum $[9,10]$. Dry skin is associated with desquamation of the stratum corneum, erythema, and itching [11]. As a transdermal patch should not be applied to the site of application where an adverse reaction has occurred, skin care is important during treatment with transdermal patches. Although moisturizers are commonly used for skin care [12], in particular, a heparinoid cream is expected to decrease skin irritation caused by the transdermal patch to a greater extent because it has high skin hydrating effects [13]. Therefore, use of heparinoid cream would decrease skin irritation caused by oxybutynin transdermal patches and improve treatment adherence. Previously, we reported that treatment with heparinoid cream before applying oxybutynin transdermal patches significantly relieved skin damage occurring after application of oxybutynin transdermal patches [14]. However, the effects of heparinoid cream in $O A B$ treatment with oxybutynin transdermal patches have not been validated clinically.

The present study was carried out to investigate the effects of a combination of heparinoid cream with an oxybutynin transdermal patch in elderly OAB patients.

\section{Methods}

\section{Study design}

This prospective observational study was performed at the Nagae Prostate Care Clinic according to the procedure depicted in Figure 1. After selecting participants based on the eligibility criteria described in the Participants subsection, heparinoid cream (Hirudoid 's soft ointment; Maruho Co., Ltd., Japan) was applied to the skin for 13 weeks. Before application of the heparinoid cream and oxybutynin transdermal patch (NEOXY TAPE 73.5 mg; Hisamitsu Pharmaceutical Co., Inc., Tokyo, Japan), skin hydration and transepidermal water loss (TEWL) were evaluated at the site of application. An oxybutynin transdermal patch was applied for 12 weeks, and its efficacy and adherence were evaluated every 2 weeks.

\section{Participants}

Study participants were selected from among $O A B$ patients of both sexes, aged $>65$ years, who consulted the Nagae Prostate Care Clinic from March to July, 2016. Patients with skin diseases; uroschesis; closed-angle glaucoma; pyloric, duodenal, or intestinal tract blockage; paralytic ileus; gastric or intestinal atony; myasthenia gravis; and those with a history of oxybutynin hydrochloride hypersensitivity were excluded.

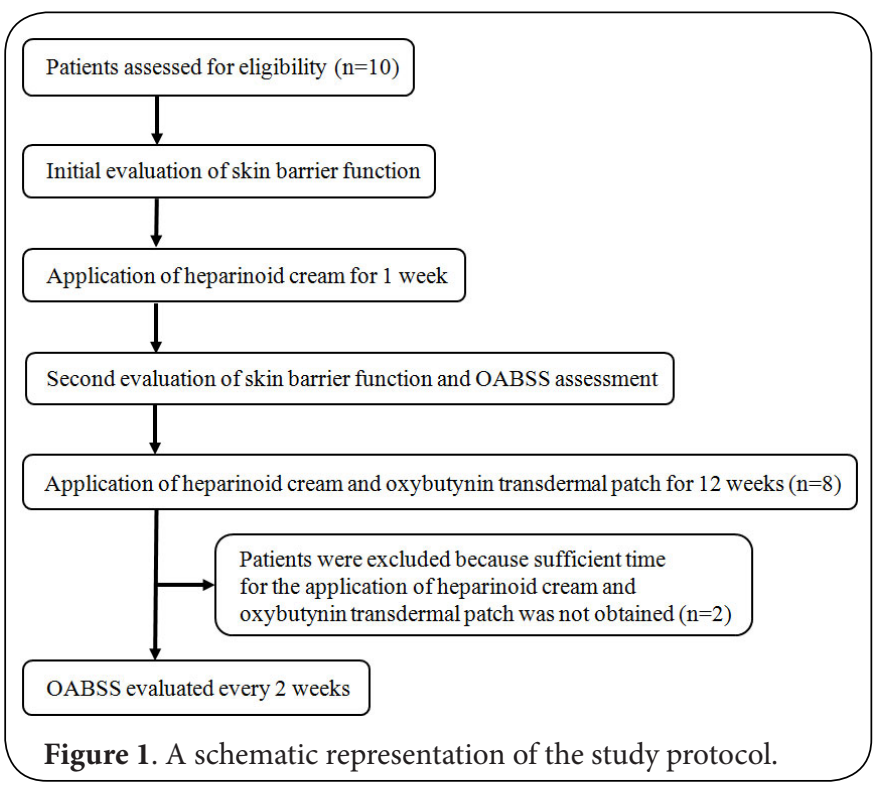

Application of heparinoid cream and oxybutynin transdermal patches

Heparinoid cream and oxybutynin transdermal patches were applied based on the schedule depicted in Figure 2. The heparinoid cream (approximately $1 \mathrm{~g}$ ) was applied twice a day for 13 weeks to the lower abdomen, femoral area, and lower back area of the participants. After 1 week, treatment with oxybutynin patches was initiated. An oxybutynin transdermal patch was applied for $24 \mathrm{~h}$ to the right side of the lower abdomen area (Figure $2\{B$ a $\}$ ) $1 \mathrm{~h}$ after application of the heparinoid cream. From the following day, the oxybutynin transdermal patch was applied consecutively to the skin areas, as illustrated in Figure 2 (B b-f).

\section{Evaluation of skin barrier function}

Skin condition in the lower abdomen, femoral area, and lower back of the participants was evaluated before application of the heparinoid cream and oxybutynin transdermal patches, as shown in Figure 2. Skin hydration and TEWL were evaluated using Corneometer CM825 and Tewameter TM300 (Courage and Khazaka Electronic DmbH, Cologne, Germany), respectively, in a room at $45-55 \%$ humidity and at $20-25^{\circ} \mathrm{C}$.

\section{Evaluation of clinical effects}

$O A B$ severity was evaluated on the basis of the OABSS, assessed every 2 weeks from the initiation of oxybutynin transdermal patch treatment (week 2). A reduction in OABSS of $>3$ points during treatment period was considered to indicate effective treatment according to the evaluation method described by Gotoh et al. [15].

\section{Statistical analysis}

All data are presented as the mean \pm standard deviation (SD) values. Statistical significance of differences between group 


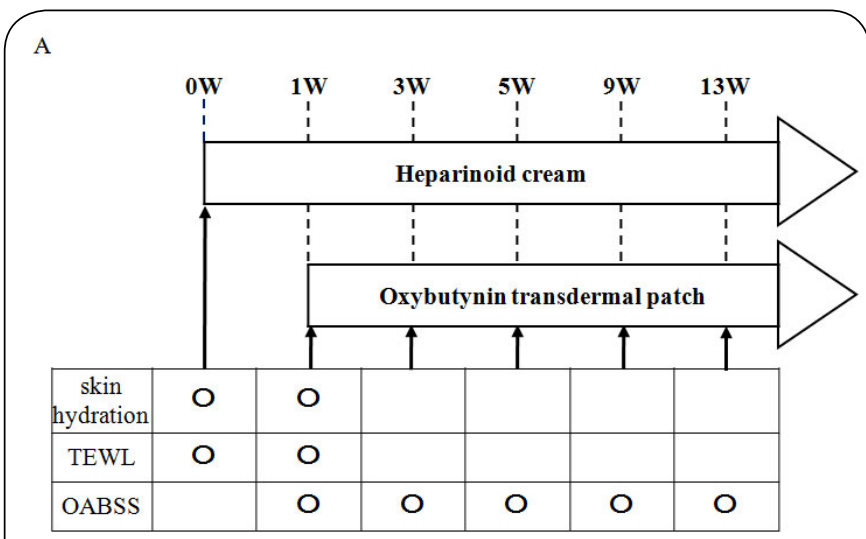

B

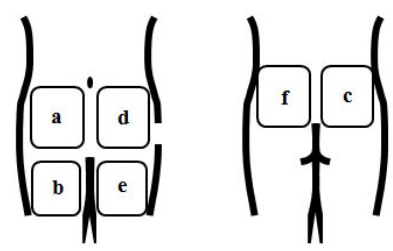

Figure 2. Schedule for the evaluation of skin barrier function and treatment with the heparinoid cream and oxybutynin transdermal patches (A) and the sites of application (B).

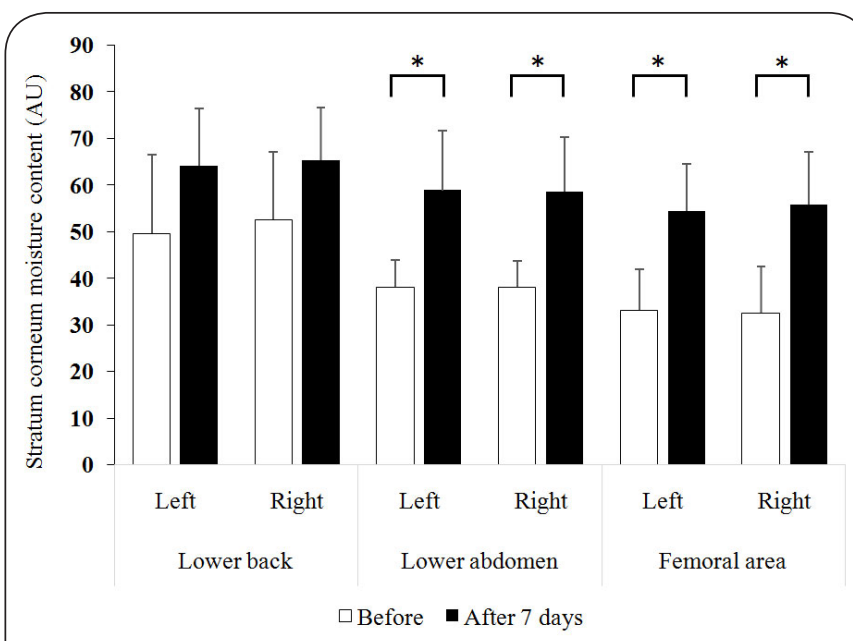

Figure 3. Effect of the heparinoid cream on moisture content in the stratum corneum Data are represented as the mean \pm standard deviation. ${ }^{\star}$ : $\mathrm{p}<0.05$ vs. before starting treatment with the heparinoid cream (Mann-Whitney U test).

means was determined using the Mann-Whitney $\mathrm{U}$ test. A $P$-value less than 0.05 indicated statistical significance.

\section{Ethics}

This study was approved by the ethics committee of Suzuka University of Medical Science (approval number: 2016-3-256) and written consent was obtained from all participants in accordance with the tenets of the Declaration of Helsinki.

\section{Results}

Effects of heparinoid cream on skin hydration and TEWL No significant differences were observed in skin hydration at the lower back area after application of the heparinoid cream; however, skin hydration in the lower abdomen and femoral areas improved significantly (Figure 3). No differences were observed in TEWL levels (Figure 4).

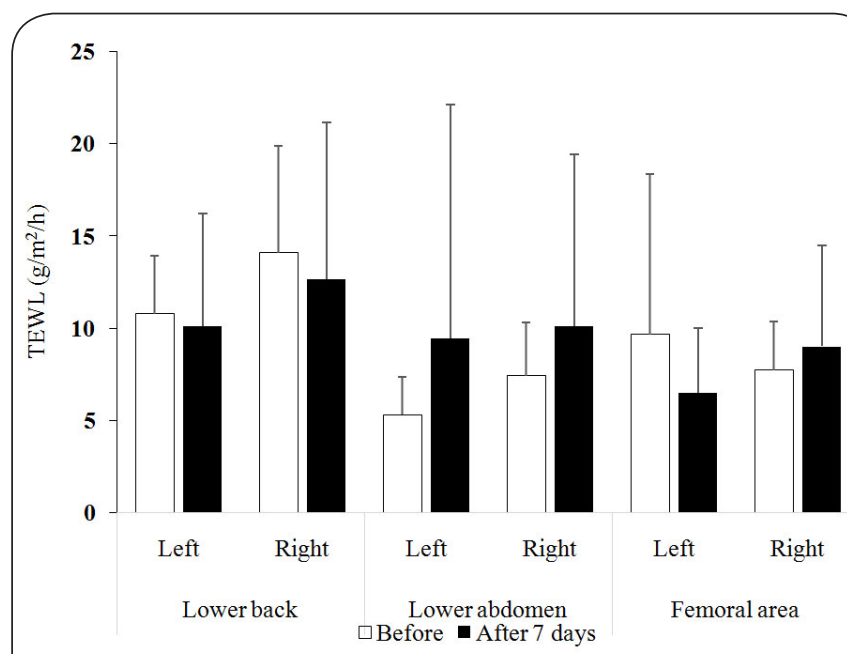

Figure 4. Effect of the heparinoid cream on transepidermal water loss Data are represented as the mean \pm standard deviation. ${ }^{*}$ : $p<0.05$ vs. before starting treatment with the heparinoid cream (Mann-Whitney U test).

Clinical effects of oxybutynin transdermal patches in OAB patients

No dermatological side effects were observed in any of the patients during treatment with the oxybutynin transdermal patch. The average OABSS decreased from 7.5 points to 4.63 points after 12 weeks of treatment (Figure 5), with 5 of 8 patients $(70 \%)$ reporting a reduction of $>3$ points in the OABSS.

\section{Discussion}

In this study, we investigated the effect of heparinoid cream application on the outcomes of treatment with an oxybutynin transdermal patch in elderly patients with $O A B$. To fulfill our study objective, 10 patients were assessed for eligible skin barrier function. Two patients were excluded because of an insufficient duration of application of the heparinoid cream and oxybutynin transdermal patch. Finally, eight patients (6 males and 2 females) were included in the study.

$O A B$ treatments using oral anticholinergic drugs decrease the quality of life of patients because of anticholinergic side effects such as dry mouth, drowsiness, and coprostasis $[16,17]$. Oxybutynin transdermal patches have a few associated anticholinergic side effects and are important in treating $O A B$ while maintaining the quality of life of patients. However, ef- 
Murakami et al. Dermatology Aspects 2018,

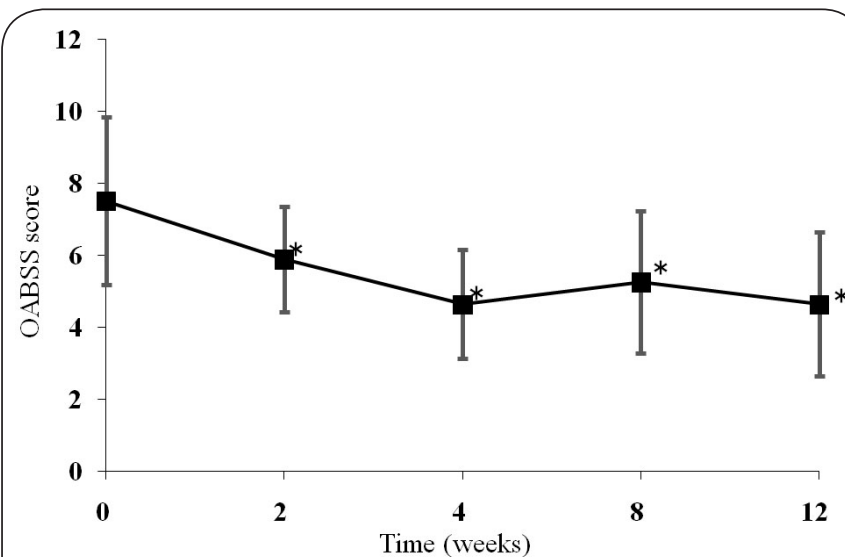

Figure 5. Time course of the overactive bladder symptom score during treatment with oxybutynin transdermal patches Data are presented as the mean \pm standard deviation. ${ }^{*}$ : $\mathrm{p}<0.05$ vs. before initiating treatment with oxybutynin transdermal patches (Mann-Whitney U test).

fective treatments with oxybutynin transdermal patches are discontinued after 3 months in approximately $64 \%$ of cases [18]. Furthermore, Yamaguchi et al. reported that mild reactions occurred at the site of application in $30.3 \%$ of elderly patients treated with an oxybutynin transdermal patch [8]. Recently, sustained application of heparinoids has effectively prevented skin irritation at the site of application of transdermal patches [19]. No oxybutynin transdermal patch-associated skin irritation was observed during this study. Our results revealed a reduction in the incidence of adverse reactions at the site of application and higher continuity rates than those reported previously. The OABSS rates four symptoms: daytime urinary frequency, nighttime urinary frequency, urgency, and urgency incontinence; a reduction in the total OABSS reflects effective $O A B$ treatment. In this study, the mean OABSS after 12-week treatment was 4.63 points. As the mean OABSS reported after the oral administration of propiverine hydrochloride and oxybutynin hydrochloride was 4.65 points [20], the therapeutic effects of the combination of the oxybutynin transdermal patch and heparinoid cream explored in this study were considered adequate. These results suggest that application of heparinoid cream effectively prolongs the period of safe use of oxybutynin transdermal patch for $O A B$ treatment without compromising therapeutic effects.

The onset of skin irritation at the site of application of transdermal patches depends on the state of the skin surface [21]. The skin of elderly individuals is easily affected by external stimuli such as cold or dry climate and use of soaps and harsh cleansers because of reduced barrier function and skin hydration caused by aging-related reduction in sebum secretion [22]. Hence, it was speculated that heparinoid cream inhibited skin irritation after application of oxybutynin transdermal patches by moisturizing the dry skin of elderly patients. However, after 1 week of heparinoid cream application,
TEWL, which is indicative of skin barrier function, remained unchanged, although skin hydration increased significantly. TEWL was previously reported to decrease after application of a heparinoid-based moisturizer [23]. However, increased TEWL was reported immediately after the application of a moisturizer because of evaporation of water in the formulation [24]. The barrier function of the stratum corneum also reportedly improved after 1 week of application of the heparinoid cream [25]. The dosing time for the eight patients was limited to 1-2 $h$ because the participants in this study were outpatients at Nagae Prostate Care Clinic and a consistent application time was required for the heparinoid cream and oxybutynin transdermal patch for all patients. Hence, the dosing interval of the medications had to be set to $1 \mathrm{~h}$, although TEWL was still quantified accurately. Therefore, high TEWL values observed in this study probably resulted from the evaporation of water from the heparinoid cream, considering that skin hydration function was improved.

Currently, the use of transdermal patches on the skin treated with creams, lotions, or powders is often not feasible owing to decreased skin adhesion. Takai et al. reported that skin adhesiveness of lidocaine tapes was not affected upon application of a heparinoid-based moisturizer [19]. Similarly, in this study, adhesion of oxybutynin transdermal patches was not affected by prior application of the heparinoid cream. Additionally, Björklund et al. reported that skin hydration increased its permeability to metronidazole [26]. Since prior application of the heparinoid cream significantly increased skin hydration in this study, transdermal absorption of oxybutynin hydrochloride may have been altered. Further fundamental animal studies evaluating transdermal absorption of oxybutynin hydrochloride after application of the heparinoid cream are required to adequately assess the proposed use of oxybutynin transdermal patches in combination with heparinoid cream.

\section{Conclusions}

Skin care using heparinoid creams has allowed for long-term treatment with oxybutynin transdermal patches without skin irritation in elderly OAB patients. This has also led to fewer side effects and prolonged treatment with stable pharmacological effects. As this technique has circumvented adverse dermatological effects associated with transdermal patches, it has potential applications in general utility for transdermal drug delivery. Drug therapy with transdermal patches has become increasingly important in recent years as the elderly population is increasing. Evaluation of the effects of moisturizers in decreasing skin irritation caused by transdermal patches plays an important role in drug treatment for elderly patients.

\section{List of Abbreviations}

DEO: N-desethyloxybutynin

OAB: overactive bladder syndrome

OABSS: overactive bladder symptom score

TEWL: transepidermal water loss 
Murakami et al. Dermatology Aspects 2018,

\section{Competing interests}

The authors declare that they have no competing interests.

\section{Authors' contributions}

\begin{tabular}{|l|c|c|c|c|c|}
\hline Authors' contributions & YM & HN & NM & HS & KO \\
\hline Research concept and design & $\checkmark$ & -- & -- & -- & $\checkmark$ \\
\hline Collection and/or assembly of data & -- & $\checkmark$ & $\checkmark$ & -- & -- \\
\hline Data analysis and interpretation & $\checkmark$ & -- & -- & $\checkmark$ & $\checkmark$ \\
\hline Writing the article & $\checkmark$ & -- & -- & $\checkmark$ & $\checkmark$ \\
\hline Critical revision of the article & $\checkmark$ & -- & -- & $\checkmark$ & $\checkmark$ \\
\hline Final approval of article & $\checkmark$ & $\checkmark$ & $\checkmark$ & $\checkmark$ & $\checkmark$ \\
\hline Statistical analysis & $\checkmark$ & -- & -- & $\checkmark$ & $\checkmark$ \\
\hline
\end{tabular}

\section{Acknowledgement}

We thank the nurse at Nagae Prostate Care Clinic for assistance in skin barrier function measurements.

Publication history

Editor: Alireza Heidari, California South University (CSU), USA. Received: 10-Jul-2018 Final Revised: 26-Aug-2018

Accepted: 30-Aug-2018 Published: 13-Sep-2018

\section{References}

1. Homma Y, Yamaguchi O and Hayashi K. Epidemiologic survey of lower urinary tract symptoms in Japan. Urology. 2006; 68:560-4. | Article | PubMed

2. Stewart WF, Van Rooyen JB, Cundiff GW, Abrams P, Herzog AR, Corey R, Hunt TL and Wein AJ. Prevalence and burden of overactive bladder in the United States. World J Urol. 2003; 20:327-36. I Article I PubMed

3. Chapple CR. Muscarinic receptor antagonists in the treatment of overactive bladder. Urology. 2000; 55:33-46; discussion 50. | Article | PubMed Abstract | PubMed FullText

4. Zobrist RH, Quan D, Thomas HM, Stanworth S and Sanders SW. Pharmacokinetics and metabolism of transdermal oxybutynin: in vitro and in vivo performance of a novel delivery system. Pharm Res. 2003; 20:103-9. I PubMed

5. Davila GW. Transdermal oxybutynin: a new treatment for overactive bladder. Expert Opin Pharmacother. 2003; 4:2315-24. | Article | PubMed

6. Appell RA, Chancellor MB, Zobrist RH, Thomas $\mathrm{H}$ and Sanders SW. Pharmacokinetics, metabolism, and saliva output during transdermal and extended-release oral oxybutynin administration in healthy subjects. Mayo Clin Proc. 2003; 78:696-702. I Article I PubMed

7. Sand P, Zinner N, Newman D, Lucente V, Dmochowski R, Kelleher C and Dahl NV. Oxybutynin transdermal system improves the quality of life in adults with overactive bladder: a multicentre, community-based, randomized study. BJU Int. 2007; 99:836-44. I Article I PubMed

8. Yamaguchi O, Uchida E, Higo N, Minami H, Kobayashi S and Sato H. Efficacy and safety of once-daily oxybutynin patch versus placebo and propiverine in Japanese patients with overactive bladder: A randomized double-blind trial. Int J Urol. 2014; 21:586-93. I Article I PubMed

9. Rogers J, Harding C, Mayo A, Banks J and Rawlings A. Stratum corneum lipids: the effect of ageing and the seasons. Arch Dermatol Res. 1996; 288:765-70. | PubMed

10. Ghadially R, Brown BE, Sequeira-Martin SM, Feingold KR and Elias PM. The aged epidermal permeability barrier. Structural, functional, and lipid biochemical abnormalities in humans and a senescent murine model. J Clin Invest. 1995; 95:2281-90. | Article | PubMed Abstract | PubMed FullText

11. Harding CR, Watkinson A, Rawlings AV and Scott IR. Dry skin, moisturization and corneodesmolysis. Int J Cosmet Sci. 2000; 22:21-52. I Article I PubMed

12. Ale I, Lachapelle JM and Maibach HI. Skin tolerability associated with transdermal drug delivery systems: an overview. Adv Ther. 2009; 26:920-35. I Article I PubMed
13. Hayama K, Takano Y, Tamura J, Tagami H and Terui T. Effectiveness of a heparinoid-containing moisturiser to treat senile xerosis. Australas J Dermatol. 2015; 56:36-9. | Article | PubMed

14. Ooi $K$, Ando $M$ and Hiramoto $K$. The research for the prevention of dermopathy to transdermal delivery systems of oxybutynin patch. Jpn Pharmacol Ther. 2015; 43:1419-1424.

15. Gotoh M, Homma Y, Yokoyama O and Nishizawa O. Responsiveness and minimal clinically important change in overactive bladder symptom score. Urology. 2011; 78:768-73. I Article I PubMed

16. Mintzer J and Burns A. Anticholinergic side-effects of drugs in elderly people. J R Soc Med. 2000; 93:457-62. I Article I PubMed Abstract | PubMed FullText

17. Scheife R and Takeda M. Central nervous system safety of anticholinergic drugs for the treatment of overactive bladder in the elderly. Clin Ther. 2005; 27:144-53. I Article I PubMed

18. Yamauti T. Usefulness of oxybutynin transdermal patch in patients with overactive bladder syndrome. J New Rem Clin. 2015; 64:33-38.

19. Takai Y, Kimura M, Matsuo H, Nomura S, Yae T and Ooi K. Influence of pre-treated moisturizer on the application of lidocaine tape for patients undergoing dialysis. Jpn J Pharm Health Care Sci. 2015; 41:855-860.

20. Homma Y, Yoshida M, Seki N, Yokoyama O, Kakizaki H, Gotoh M, Yamanishi T, Yamaguchi O, Takeda M and Nishizawa O. Symptom assessment tool for overactive bladder syndrome--overactive bladder symptom score. Urology. 2006; 68:318-23. I Article I PubMed

21. Darlenski R, Kazandjieva J, Tsankov N and Fluhr JW. Acute irritant threshold correlates with barrier function, skin hydration and contact hypersensitivity in atopic dermatitis and rosacea. Exp Dermatol. 2013; 22:752-3. I Article I PubMed

22. Rawlings $A V$ and Harding $C R$. Moisturization and skin barrier function. Dermatol Ther. 2004; 17 Suppl 1:43-8. I PubMed

23. Ishii R, Kataoka M, Hosokawa S, Doi T, Tobetto K, Hirano T, Enomoto A, Aki $\mathrm{H}$ and Naruse T. Mechanisms of heparinoid-induced skin moisturizing effect influence on natural moisturizing factors. Nishinihon J Dermatol. 2007; 69:51-56.

24. Loden $M$. The increase in skin hydration after application of emollients with different amounts of lipids. Acta Derm Venereol. 1992; 72:327-30. I PubMed

25. Kawashima M and Ishizaki C. Evaluation of the efficacy of medical moisturizers on artificially dried skin and atopic dry skin on the basis of dermato-physiological parameters. Jpn J Derm. 2007; 117:275-284.

26. Bjorklund S, Engblom J, Thuresson K and Sparr E. Glycerol and urea can be used to increase skin permeability in reduced hydration conditions Eur J Pharm Sci. 2013; 50:638-45. | Article | PubMed

Citation:

Murakami Y, Nagae H, Maehori N, Sekijima H and Ooi K. Combination of oxybutynin transdermal patch and heparinoid cream for long-term treatment of overactive bladder in elderly patients. Dermatol Aspects. 2018; 6:1.

http://dx.doi.org/10.7243/2053-5309-6-1 